with idiopathic ataxia, but recent studies have indicated that they are also found in systemic lupus erythematosus (SLE). The goals of this study were to determine the frequency of anti-MPP-1 in a local SLE cohort and then identify demographic, clinical, and serologic correlations.

Methods Patients fulfilling the American College of Rheumatology (ACR) or Systemic Lupus International Collaborating Clinics (SLICC) Classification Criteria for SLE were enrolled in the SouThern Alberta Registry for Lupus EryThematosus (STARLET) cohort (Calgary, Canada). Demographic, clinical information (disease activity - SLEDAI-2K; damage - SLICC/ ACR DI), and sera were collected at time of enrollment. Antibodies to MPP-1 were determined by an addressable laser bead immunoassay (ALBIA) utilizing an in vitro expressed MPP-1 cDNA construct inserted into a GFP vector (Clontech Laboratories Inc., Saint-Germain-en-Laye, France). ALBIA results were expressed as median florescence units (MFU) and a dilution of $\geq 1: 500$ MFU was considered highly positive. Univariable analysis was performed to determine associations between the prevalence of high positive anti-MPP-1 and demographic (age, sex, race/ethnicity), clinical features (SLEDAI-2K and SLICC/ACR DI total scores and subscales and neurological subscale of the ACR and SLICC Classification Criteria), medications, other autoantibodies (anti-dsDNA, extractable nuclear antigens, and anti-phospholipid antibodies).

Results One hundred and forty SLE patients were included; $89.3 \%$ were female with a mean age of 47.3 years (SD 16.3) and disease duration of 13.9 years (SD 11.6). The prevalence of high titre anti-MPP-1 was 15.0\% (21/140) respectively. Univariable analysis demonstrated that high anti-MPP-1 positivity was associated with a higher total SLEDAI-2K score (Odds Ratio (OR), 1.1 [95\% CI 1.0 to 1.3]), particularly with the serositis (OR 3.0, [95\% CI 1.4 to 6.6]) and immunological subscales (OR 2.0, [95\% CI 1.4 to 2.9]). High anti-MPP-1 positivity was also associated with anti-dsDNA (OR 5.5 [95\% CI 1.8 to 16.6]), anti-SSA/Ro60 (OR 3.1 [95\% CI 1.0 to 8.9]) and anti-phosphotidylserine/prothrombin complex (aPS/ PT)-IgG (OR 3.6 [95\% CI 1.1 to 11.5]).

Conclusions High titer anti-MPP-1 antibodies were common in this SLE cohort (15.0\%) and may be associated with greater clinical and serologic SLE disease activity. A larger study is currently underway to more clearly delineate its role as a biomarker in SLE.

Acknowledgements The authors are grateful for the technical assistance of Ms. Haiyan Hou and Meifeng Zhang (Mitogen Advanced Diagnostics, University of Calgary).

\section{AA-05 B CELL INTRINSIC IFN $\beta$ IS ASSOCIATED WITH AUTOANTIBODIES AND ACTIVE RENAL DISEASE IN SLE}

${ }^{1}$ W Winn Chatham*, 'Jennie A Hamilton, 'Qi Wu, ${ }^{1}$ PingAr Yang, ${ }^{1}$ Bao Luo, 'Shanrun Liu, ${ }^{1}$ Jun Li, ${ }^{1}$ Hui-Chen Hsu, ${ }^{2}$ Inaki Sanz, ${ }^{3}$ John D Mountz. 'Department of Medicine, University of Alabama at Birmingham, Birmingham, AL; ${ }^{2}$ Department of Medicine, Emory University; ${ }^{3}$ Department of Medicine, Birmingham VA Medical Center, Birmingham

\subsection{6/lupus-2018-Ism.24}

Background Dysregulated responses to type I interferons (IFNs) is a hallmark of autoreactive B cell development in SLE patients. High sera levels of type I IFN protein were recently shown to occur in the absence of increased circulating pDCs and in the absence of increased pDC IFNs, suggesting the likelihood of other important sources of type I IFN that may act on B cells. The present study determined the cellular source of IFN $\beta$ and its association with disease activities in SLE.

Methods Peripheral blood mononuclear cells (PBMCs) were obtained from 31 SLE patients meeting ACR 1997 revised criteria for SLE and 9 healthy controls. Intracellular IFN $\beta$ was determined using flow cytometry with FITC-anti-IFN $\beta$ mAb. Comprehensive clinical data was recorded for each SLE subject and the clinical data and laboratory analysis of B-cell intracellular IFN $\beta$ expression were collected in a double-blind fashion.

Results IFN $\beta$ was detected in various cell types including CD4 $\mathrm{T}$ cells, B cells and pDCs in PBMCs of SLE patients. Endogenous IFN $\beta$ in $B$ cells was significantly higher than endogenous IFN $\beta$ in CD4 $T$ cells and were equivalent to that seen in pDCs. Within B cells, there was a significant increase in endogenous IFN $\beta$ in all B cell subpopulations of SLE patients compared to healthy controls. The most significant increase was found in the $\mathrm{CD} 27^{+} \mathrm{IgD}^{-}$memory subpopulation. B-cell endogenous IFN $\beta$ was not a result of B-cell uptake of exogenous IFN $\beta$ as coculture of SLE B cells with HEK293 reporter cells resulted in induction of interferon stimulatory genes as determined by the secreted alkaline phosphatase assay. This was further blocked by an anti-IFN $\beta$ neutralization antibody. Interestingly B-cell endogenous IFN $\beta$ was highly correlated with clinical disease including renal disease and autoantibodies including anti-dsDNA, anti-Sm and anti-SSA. Strikingly, the highest correlation of IFN $\beta$ with clinical manifestations was observed in African-American patients. B-cell IFN $\beta$ expression was significantly correlated with $\mathrm{CD} 19^{\mathrm{lo}} \mathrm{CD} 38^{\text {hi }} \mathrm{CD} 27^{+}$plasma cell formation.

Conclusion Intracellular IFN $\beta$ production by $\mathrm{B}$ cells is a novel and important B-cell intrinsic factor that may be essential for B-cell development into autoantibody producing B cells. The present work suggests a need for future human lupus studies into type I IFN dysregulation that pioneer beyond the view of pDC produced IFN $\alpha$. These results also provided a mechanistic basis for development of more effective therapies to target the high-affinity IFN $\beta$ or the enhanceosome components that promote its induction in a subgroup of lupus patients.

Acknowledgements This work was supported by grants from R01-AI-071110, R01 AI134023, Lupus Research Alliance Distinguished Innovator Award, I01B $\times 004049$, and $1 \mathrm{I} 01 \mathrm{~B} \times$ 000600 to J.D.M, 2T32AI007051-39 Immunology T32 Training Grant and the LFA Finzi Summer Fellowship to J.A.H, and the LRA Novel Research Award to H.-C.H.

\section{Big Data Analyses}

\section{BD-01 E-GENES IDENTIFIED VIA TRANSANCESTRAL SNP MAPPING AND GENE EXPRESSION ANALYSIS REVEAL NOVEL TARGETED THERAPIES FOR AFRICAN-AMERICAN AND EUROPEAN-AMERICAN SLE PATIENTS}

${ }^{1}$ Katherine A Owen, ${ }^{1}$ Bryce N Aidukaitis, ${ }^{1}$ Adam C Labonte, ${ }^{1}$ Michelle D Catalina, ${ }^{1}$ Prathyusha Bachali, ${ }^{1} J a m e s$ Dittman, ${ }^{1}$ Nicholas Geraci, ${ }^{1}$ Sean Rouffa, ${ }^{2}$ Hannah C Ainsworth, ${ }^{2}$ Miranda C Marion, ${ }^{2}$ Timothy D Howard, ${ }^{2}$ Carl D Langefeld, ${ }^{1}$ Peter E Lipsky, ${ }^{1}$ Amrie C Grammer*. ${ }^{1}$ RILITE Research Institute, Charlottesville, VA, USA; ${ }^{2}$ Wake Forest School of Medicine, Winston-Salem, NC, USA

\subsection{6/lupus-2018-Ism.25}

Background Systemic lupus erythematosus (SLE) in AfricanAmericans (AA) is more prevalent, more severe and associated 


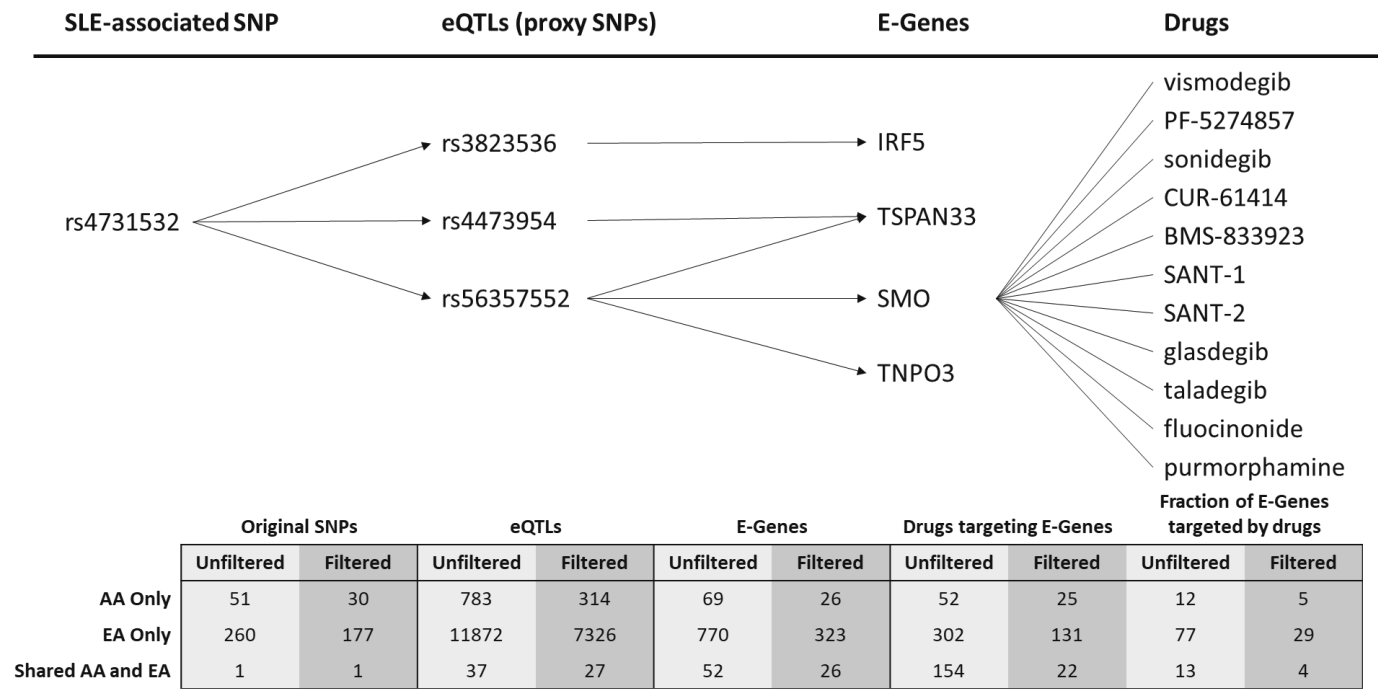

Abstract BD-01 Figure 1 Unpacking an SLE-associated SNP

with an increased burden of co-morbidities compared to European-American (EA) populations. Genome-wide association studies (GWAS) have linked many single nucleotide polymorphisms (SNPs) to SLE. Recently, Langefeld (2017) conducted a large-scale transancestral association study of SLE to identify ancestry-dependent and independent contributions to SLE risk. We extend these findings to include a transancestral analysis linking SLE-associated SNPs to candidate-causal E-Genes specific to AA and EA populations and differential gene expression in these populations with the goal of matching genetic and genomic disease characteristics with available treatments unique to each ancestral group.

Methods SNPs proxy to SLE-associated SNPs were compared with known expression quantitative trait loci (eQTLs) contained in the GTEx (version 6) database. E-QTLs and their associated E-Genes were divided by ancestry and compared to differentially expressed (DE) genes from multiple SLE gene expression datasets. For both ancestral groups, E-Gene lists were examined for the significant enrichment of BIG-C categories and IPA (Qiagen) Canonical Pathways to predict novel upstream regulators (UPRs). For visualization and clustering analysis, STRING-generated networks of DE E-Genes were imported into Cytoscape (version 3.6.1) and partitioned with the community clustering (GLay) algorithm via the clusterMaker2 (version 1.2.1) plugin. Finally, drug candidates targeting E-Genes, DE genes and UPRs were identified using CLUE, REST, API, IPA and STITCH (version 5.0; http://stitch.embl. de). The process of unpacking an SLE-associated SNP is shown in figure 1.

Results E-QTL and DE gene queries of GTEx were combined and newly predicted E-Genes were pooled by ancestry. Here, we identify 52 SNPs with eQTLs unique to AA ancestry, 260 SNPs unique to EA ancestry and 1 SNP shared between ancestries. Together, these SNPs identified a total of 891 distinct EGenes associated with both ancestral groups. In studies comparing E-Genes to SLE DE data sets, 516 EA E-Genes were differential expressed compared to 48 AA E-Genes. Comparison with various drug candidate databases resulted in the identification of 12 drugs targeting genes specific for AA, 77 drugs specific for EA genes and 13 shared between EA and AA. Predicted EA-specific drugs include hydroxychloroquine and drugs-in-development targeting CD40LG, CXCR1 and CXCR2 whereas AA-specific drugs include HDAC inhibitors, retinoids, and drugs targeting IRAK4 and CTLA4. Drugs targeting EGenes/pathways shared by EA and AA include ibrutinib, ruxolitinib and ustekinumab.

Conclusions The ancestral SNP-associated E-Genes and gene expression profiles outlined here illustrate fundamental differences in lupus molecular pathways between AA and EA. Our results indicate that unique sets of drugs may be particularly effective at treating lupus within each ancestral group.

Acknowledgments Financial support for this research was provided by RILITE Research Institute.

\section{BD-02 BLOCKADE OF THE MECHANISTIC TARGET OF RAPAMYCIN ELICITS RAPID AND LASTING IMPROVEMENT OF DISEASE ACTIVITY THROUGH RESTRAINING PRO-INFLAMMATORY T CELL LINEAGE SPECIFICATION IN PATIENTS WITH ACTIVE SLE}

Zhi-Wei Lai, Ryan Kelly, Thomas Winans, Ivan Marchena, Ashwini Shadakshari, Julie Yu, Maha Dawood, Ricardo Garcia, Hajra Tily, Lisa Francis, Stephen V Faraone, Paul E Phillips, Andras Perl*. Division of Rheumatology, Departments of Medicine, Microbiology and Immunology, and Biochemistry and Molecular Biology, State University of New York, Upstate Medical University, College of Medicine, Syracuse, New York

\subsection{6/lupus-2018-Ism.26}

Background Systemic lupus erythematosus (SLE) patients exhibit T-cell dysfunction that has been attributed to mechanistic target of rapamycin activation. Therefore, safety, tolerance, and efficacy of rapamycin were examined in a prospective biomarker-driven open-label clinical trial.

Methods 40 patients having active disease and unresponsive or intolerant to conventional medications were enrolled. Sirolimus was started at $2 \mathrm{mg} /$ day with dosage adjusted to tolerance and 6-15 ng/ml trough levels. Disease activity was evaluated by BILAG, SLEDAI, and prednisone use over 12 months. Blood samples of 56 matched healthy subjects were obtained as controls for immunometabolic outcomes monitored at each visit. Results 11 patients dropped out, 9 for non-compliance and 2 for intolerance. Among safety outcomes, liver function and lymphocyte counts were unchanged. While HDL-cholesterol, neutrophil counts and haemoglobin were moderately reduced, 